\title{
Editorial
}

\section{Joint Models and Their Applications}

\author{
Yangxin Huang, ${ }^{1}$ Lang $\mathrm{Wu}{ }^{2}$ Grace $\mathrm{Y} . \mathrm{Yi}^{3}{ }^{3}$ and Wenbin $\mathrm{Lu}^{4}$ \\ ${ }^{1}$ Department of Epidemiology \& Biostatistics, College of Public Health, University of South Florida, \\ Tampa, FL 33612, USA \\ ${ }^{2}$ Department of Statistics, University of British Columbia, Vancouver, BC, Canada V6T 1Z2 \\ ${ }^{3}$ Department of Statistics and Actuarial Science, University of Waterloo, Waterloo, ON, Canada N2L 3G1 \\ ${ }^{4}$ Department of Statistics, North Carolina State University, Raleigh, NC 27695, USA
}

Correspondence should be addressed to Yangxin Huang, yhuang@health.usf.edu

Received 27 December 2011; Accepted 27 December 2011

Copyright (c) 2012 Yangxin Huang et al. This is an open access article distributed under the Creative Commons Attribution License, which permits unrestricted use, distribution, and reproduction in any medium, provided the original work is properly cited.

A common objective in longitudinal studies is to characterize the relationship between a longitudinal response process and a time to event. Considerable interest has been focused on the so-called joint models, where models for the event time distribution and longitudinal data are often specified through a common set of latent random effects. Joint models of longitudinal data and/or survival data have received great attention in the literature over the past two decades and are becoming increasingly active area of statistics research. The importance of these models is well recognized, partly due to the fact that longitudinal data and survival data arise frequently in practice. Despite the extensive literature on this topic, these models continue to be a main research stream since they offer many advantages over separate analysis of longitudinal data and/or survival data. To accelerate the development of advanced tools and knowledge of joint models, a number of important issues remain to be addressed such as computational issues, model diagnostics and selections, joint models with skew distributions, and various choices of longitudinal models and survival models. To stimulate the continuing efforts to understand various joint model development and associated statistical inference methods with their applications in biomedical, biological, engineering, and other studies, in this special issue, we have invited a few papers that address some of those issues.

The paper by L. Wu et al., in this special issue, provides a brief overview of various formulations of joint models for longitudinal and survival data. Commonly used methods including the likelihood method and two-stage methods are discussed in detail, and other joint modeling methods such as Bayesian approach are also briefly presented. Computational issues are investigated. A real data analysis and a simulation study are provided to compare the performance of various methods. The paper by M. Liu and W. Lu delivers a semiparametric marginal inference approach for longitudinal outcomes in the presence 
of informative dropouts. The dependence between longitudinal outcome and informative dropout time is characterized by a conditional mean model, and the longitudinal regression coefficients are estimated by a class of conditional generalized estimating equations. The proposed method is robust against a class of latent-variable models for longitudinal data with informative dropouts and is computationally easy to implement. G. A. Dagne and Y. Huang's paper presents joint Tobit models for a left-censored response variable with skewness and covariate variables with substantial measurement errors. The proposed joint models are a skew- $t$ nonlinear mixed-effects Tobit model for the response process and a skew$t$ nonparametric mixed-effects model for covariate process under a Bayesian framework. A real data example is used to illustrate the proposed methods. The paper by M. Murawska et al. proposes a two-stage approach that summarizes the longitudinal information with nonlinear mixed-effects model at the first stage, and includes empirical Bayes estimates of the subject-specific parameters as predictors in the Cox model for time-to-event at the second stage. To take into account the uncertainty of the estimated subject-specific parameters, the authors use a Monte Carlo approach and sample from the posterior distribution of the random effects given the observed data. The paper by S. Gurmu and G. A. Dagne, in this special issue, develops zero-inflated bivariate-ordered probit model and carries out estimation using Markov Chain Monte Carlo techniques in the context of Bayesian analysis of a joint model of ordered outcomes. The authors analyze the socioeconomic determinants of individual problem of smoking and chewing tobacco using household tobacco survey data with substantial proportion of zeros. The example shows that the use of a model that ignores zero-inflation masks differential effects of covariates on nonusers and users.

Yangxin Huang

Lang $W u$

Grace Y. Yi

Wenbin Lu 


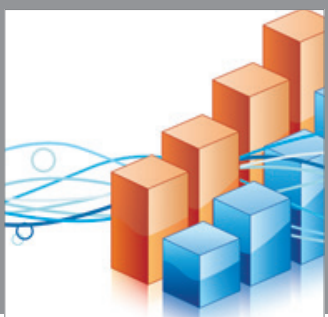

Advances in

Operations Research

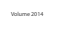

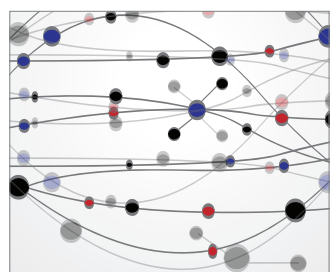

\section{The Scientific} World Journal
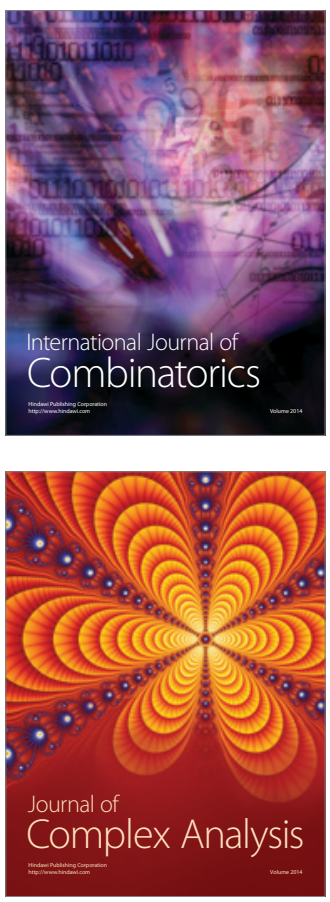

International Journal of

Mathematics and

Mathematical

Sciences
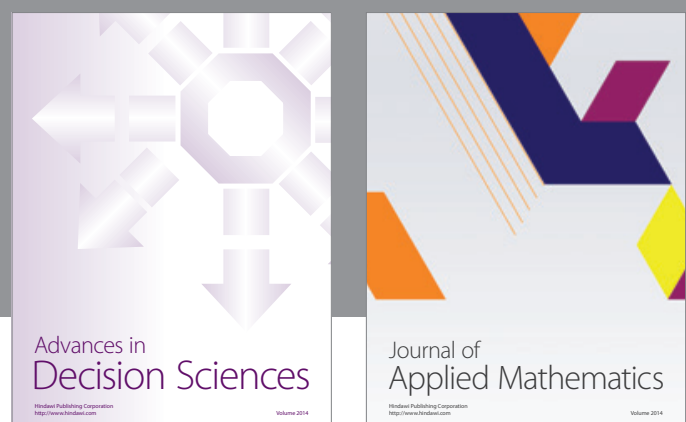

Journal of

Applied Mathematics
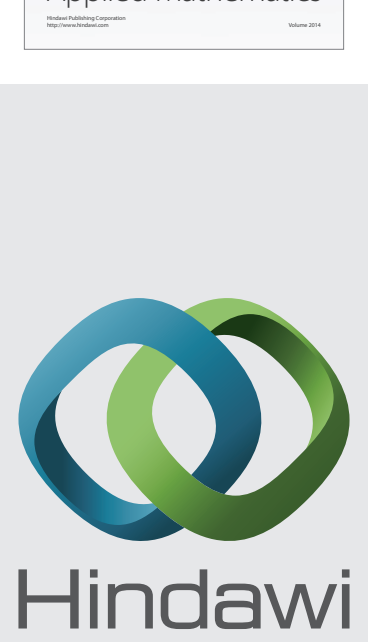

Submit your manuscripts at http://www.hindawi.com
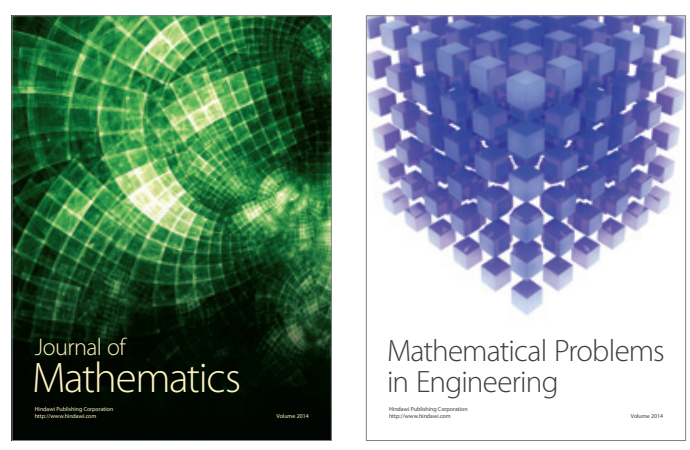

Mathematical Problems in Engineering
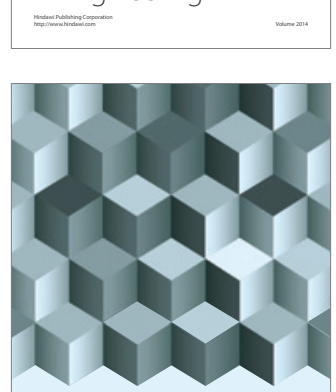

Journal of

Function Spaces
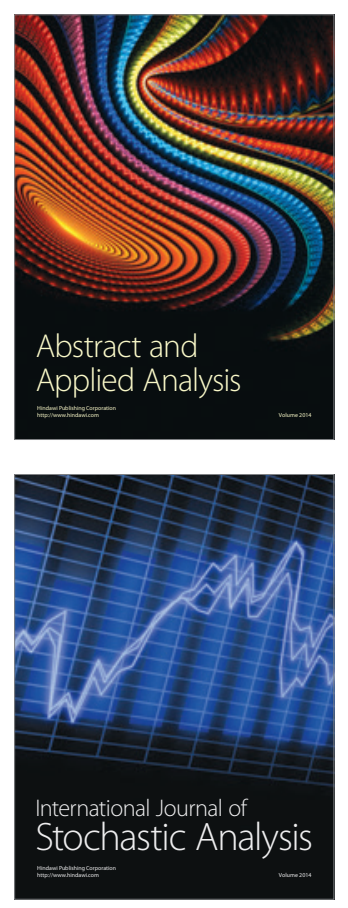

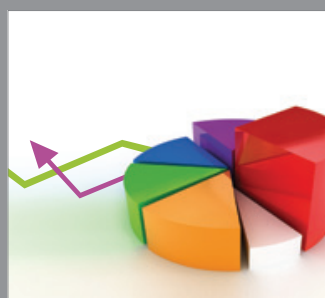

ournal of

Probability and Statistics

Promensencen
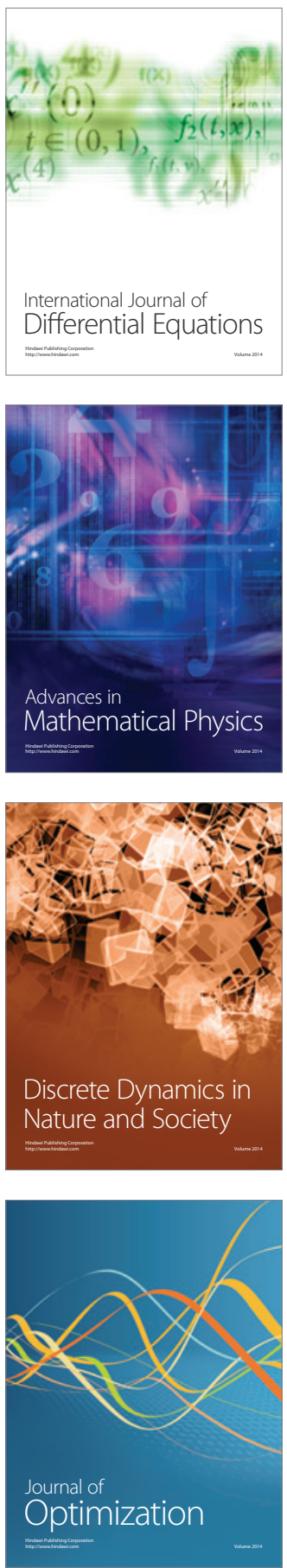